\title{
Dalitz Plot Analysis of Three-body Charmonium Decays at BABAR
}

\author{
Antimo Palano ${ }^{1,3, \star}$ \\ ${ }^{1}$ INFN and University of Bari, Via Orabona 4, 70125 Bari, Italy
}

\begin{abstract}
We present results on the measurement of the $\mathrm{I}=1 / 2 K \pi \mathcal{S}$-wave through a model independent partial wave analysis of $\eta_{c}$ decays to $K_{S}^{0} K^{+} \pi^{-}$and $K^{+} K^{-} \pi^{0}$ produced in two-photon interactions. We also perform Dalitz plot analyses of the $J / \psi$ decays to $\pi^{+} \pi^{-} \pi^{0}, K^{+} K^{-} \pi^{0}$ and $K_{S}^{0} K^{ \pm} \pi^{\mp}$ produced in the initial state radiation process.
\end{abstract}

\section{Introduction}

Charmonium decays can be used to obtain new information on light meson spectroscopy. In $e^{+} e^{-}$ interactions, samples of charmonium decays can be obtained using different processes.

- In two-photon interactions we select events in which the $e^{+}$and $e^{-}$beam particles are scattered at small angles and remain undetected. Only resonances with $J^{P C}=0^{ \pm+}, 2^{ \pm+}, 3^{++}, 4^{ \pm+} \ldots$ can be produced.

- In the Initial State Radiation (ISR) process, we reconstruct events having a (mostly undetected) fast forward $\gamma_{I S R}$ and, in this case, only $J^{P C}=1^{--}$states can be produced.

\section{Study of $\eta_{c} \rightarrow K \bar{K} \pi$}

The BaBar Dalitz plot analysis of the $\eta_{c} \rightarrow K^{+} K^{-} \eta$ and $\eta_{c} \rightarrow K^{+} K^{-} \pi^{0}$ has provided the unexpected observation of $K_{0}^{*}(1430) \rightarrow K \eta[1]$. We also find that the $\eta_{c}$ three-body hadronic decays proceed almost entirely through the intermediate production of scalar meson resonances.

We study the reactions [2]

$$
\gamma \gamma \rightarrow K_{s}^{0} K^{+} \pi^{-}, \quad \gamma \gamma \rightarrow K^{+} K^{-} \pi^{0} .
$$

In the following, details on events reconstruction will be given only for the $K_{S}^{0} K^{+} \pi^{-}$final state. We select events having only four tracks. Since two-photon events balance the transverse momentum, we require $p_{T}$, the transverse momentum of the $K_{S}^{0} K^{+} \pi^{-}$system with respect to the beam axis, to be $p_{T}<0.08 \mathrm{GeV} / \mathrm{c}$. We also define $M_{\mathrm{rec}}^{2} \equiv\left(p_{e^{+} e^{-}}-p_{\mathrm{rec}}\right)^{2}$, where $p_{e^{+} e^{-}}$is the four-momentum of the initial state and $p_{\text {rec }}$ is the four-momentum of the $K_{s}^{0} K^{+} \pi^{-}$system and remove ISR events requiring $M_{\text {rec }}^{2}>10 \mathrm{GeV}^{2} / \mathrm{c}^{4}$. The $K_{s}^{0} K^{+} \pi^{-}$mass spectrum in the $\eta_{c}$ mass regions is shown in Fig. 1(left) and the $\eta_{c}$ Dalitz plot is shown in Fig. 1(right). The Dalitz plot is dominated by the presence of horizontal and vertical uniform bands at the position of the $K_{0}^{*}(1430)$ resonance. The corresponding distributions for $\eta_{c} \rightarrow K^{+} K^{-} \pi^{0}$ can be found in Ref. [1] and present similar features.

^e-mail: antimo.palano@ba.infn.it 

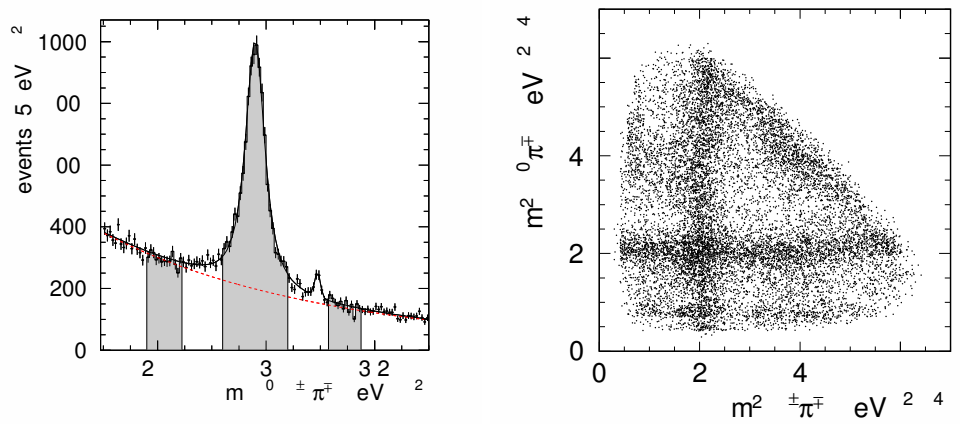

Figure 1. (left) $K_{S}^{0} K^{+} \pi^{-}$mass spectrum in two-photon interactions and (right) the $\eta_{c} \rightarrow K_{S}^{0} K^{+} \pi^{-}$Dalitz plot.

The $\eta_{c}$ signal regions contain 12849 events with $(64.3 \pm 0.4) \%$ purity for $\eta_{c} \rightarrow K_{s}^{0} K^{+} \pi^{-}$and 6494 events with $(55.2 \pm 0.6) \%$ purity for $\eta_{c} \rightarrow K^{+} K^{-} \pi^{0}$. The backgrounds below the $\eta_{c}$ signals are estimated from the sidebands. We observe asymmetric $K^{*}$ 's in the background to the $\eta_{c} \rightarrow K_{S}^{0} K^{+} \pi^{-}$ final state due to interference between $\mathrm{I}=1$ and $\mathrm{I}=0$ contributions.

\section{Dalitz plot Analysis of $\eta_{c} \rightarrow K \bar{K} \pi$}

We perform unbinned maximum likelihood fits using the Isobar model [3] and Model Independent Partial Wave Analysis (MIPWA) [4]. In the MIPWA the $K \pi$ mass spectrum is divided into 30 equally spaced mass intervals $60 \mathrm{MeV} / c^{2}$ wide and for each bin we add to the fit two new free parameters, the amplitude and the phase of the $K \pi \mathcal{S}$-wave (constant inside the bin). We also fix the amplitude to 1.0 and its phase to $\pi / 2$ in an arbitrary interval of the mass spectrum (bin 11 which corresponds to a mass of $1.45 \mathrm{GeV} / c^{2}$ ). The number of additional free parameters is therefore 58 . Due to isospin conservation in the decays, amplitudes are symmetrized with respect to the two $K \pi$ decay modes. The $K_{2}^{*}(1420), a_{0}(980), a_{0}(1400), a_{2}(1310), \ldots$ contributions are modeled as relativistic Breit-Wigner functions multiplied by the corresponding angular functions. Backgrounds are fitted separately and interpolated into the $\eta_{c}$ signal regions. The fits improves when an additional high mass $a_{0}(1950) \rightarrow$ $K \bar{K} \mathrm{I}=1$ resonance is included with free parameters in both $\eta_{c}$ decay modes. The weighted average of the two measurement is: $m\left(a_{0}(1950)\right)=1931 \pm 14 \pm 22 \mathrm{MeV} / c^{2}, \Gamma\left(a_{0}(1950)\right)=271 \pm 22 \pm 29 \mathrm{MeV}$. The statistical significances for the $a_{0}(1950)$ effect (including systematics) are $2.5 \sigma$ for $\eta_{c} \rightarrow K_{S}^{0} K^{+} \pi^{-}$ and $4.0 \sigma$ for $\eta_{c} \rightarrow K^{+} K^{-} \pi^{0}$.

The Dalitz plot projections with fit results for $\eta_{c} \rightarrow K_{S}^{0} K^{+} \pi^{-}$and $\eta_{c} \rightarrow K^{+} K^{-} \pi^{0}$ are shown in Fig. 2. The fitted fractions and phases are given in Table 1. We observe a good description of the data. We note that the $K^{*}(892)$ contributions arise entirely from background.

In comparison, the isobar model gives a worse description of the data, with $\chi^{2} / N_{\text {cells }}=457 / 254=$ 1.82 and $\chi^{2} / N_{\text {cells }}=383 / 233=1.63$ respectively for the two $\eta_{c}$ decay modes. The resulting $K \pi$ $\mathcal{S}$-wave amplitude and phase for the two $\eta_{c}$ decay modes is shown in Fig. 3. We observe a clear $K_{0}^{*}(1430)$ resonance signal with the corresponding expected phase motion. At high mass we observe the presence of the broad $K_{0}^{*}(1950)$ contribution with good agreement between the two $\eta_{c}$ decay modes. Comparing with LASS [5] and E791 [4] experiments we note that phases before the $K \eta^{\prime}$ threshold are similar, as expected from Watson [6] theorem but amplitudes are very different. 

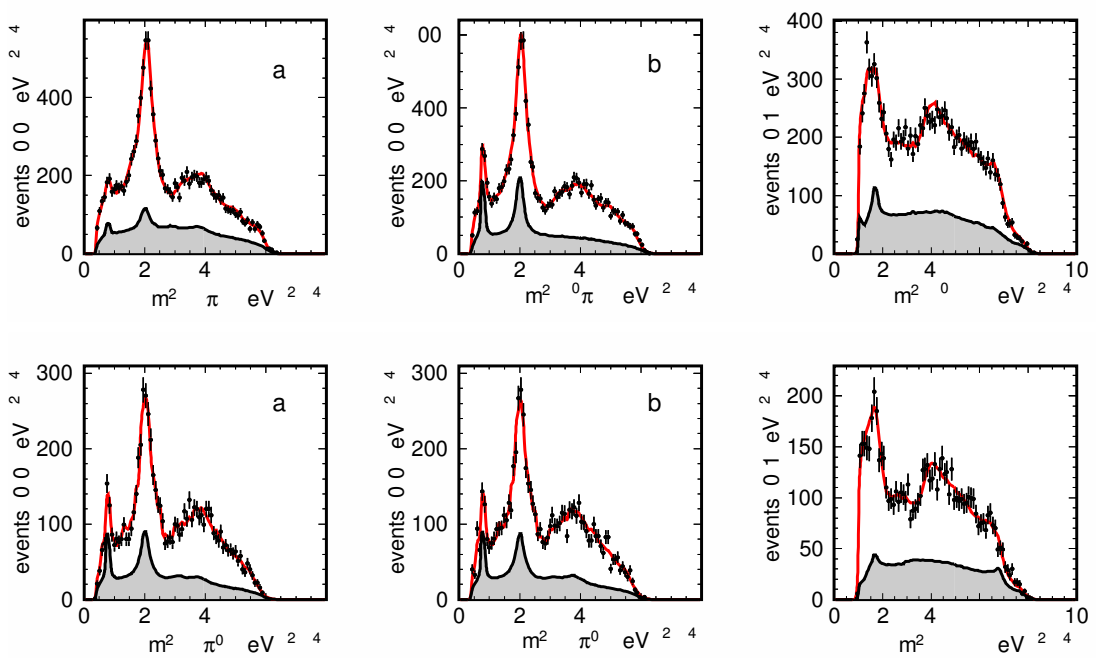

Figure 2. (top) $\eta_{c} \rightarrow K_{S}^{0} K^{+} \pi^{-}$and (bottom) $\eta_{c} \rightarrow K^{+} K^{-} \pi^{0}$ Dalitz plots projections. The superimposed curves are from the fit results. Shaded is contribution from the interpolated background.

Table 1. Results from the $\eta_{c} \rightarrow K_{S}^{0} K^{ \pm} \pi^{\mp}$ and $\eta_{c} \rightarrow K^{+} K^{-} \pi^{0}$ MIPWA. Phases are determined relative to the ( $K \pi \mathcal{S}$-wave) $\bar{K}$ amplitude which is fixed to $\pi / 2$ at $1.45 \mathrm{GeV} / c^{2}$.

\begin{tabular}{|l|r|r|r|r|}
\hline & \multicolumn{2}{|c|}{$\eta_{c} \rightarrow K_{S}^{0} K^{ \pm} \pi^{\mp}$} & \multicolumn{2}{c|}{$\eta_{c} \rightarrow K^{+} K^{-} \pi^{0}$} \\
\hline Amplitude & \multicolumn{1}{|c|}{ Fraction (\%) } & \multicolumn{1}{c|}{ Phase (rad) } & \multicolumn{1}{|c|}{ Fraction (\%) } & \multicolumn{1}{c|}{ Phase (rad) } \\
\hline$(K \pi \mathcal{S}$-wave) $\bar{K}$ & $107.3 \pm 2.6 \pm 17.9$ & fixed & $125.5 \pm 2.4 \pm 4.2$ & fixed \\
$a_{0}(980) \pi$ & $0.8 \pm 0.5 \pm 0.8$ & $1.08 \pm 0.18 \pm 0.18$ & $0.0 \pm 0.1 \pm 1.7$ & - \\
$a_{0}(1450) \pi$ & $0.7 \pm 0.2 \pm 1.4$ & $2.63 \pm 0.13 \pm 0.17$ & $1.2 \pm 0.4 \pm 0.7$ & $2.90 \pm 0.12 \pm 0.25$ \\
$a_{0}(1950) \pi$ & $3.1 \pm 0.4 \pm 1.2$ & $-1.04 \pm 0.08 \pm 0.77$ & $4.4 \pm 0.8 \pm 0.8$ & $-1.45 \pm 0.08 \pm 0.27$ \\
$a_{2}(1320) \pi$ & $0.2 \pm 0.1 \pm 0.1$ & $1.85 \pm 0.20 \pm 0.20$ & $0.6 \pm 0.2 \pm 0.3$ & $1.75 \pm 0.23 \pm 0.42$ \\
$K_{2}^{*}(1430) \bar{K}$ & $4.7 \pm 0.9 \pm 1.4$ & $4.92 \pm 0.05 \pm 0.10$ & $3.0 \pm 0.8 \pm 4.4$ & $5.07 \pm 0.09 \pm 0.30$ \\
\hline Total & $116.8 \pm 2.8 \pm 18.1$ & & $134.8 \pm 2.7 \pm 6.4$ & \\
$-2 \log \mathcal{L}$ & -4314.2 & & -2339 & \\
$\chi^{2} / N_{\text {cells }}$ & $301 / 254=1.17$ & & $283.2 / 233=1.22$ & \\
\hline
\end{tabular}

\section{Dalitz plot analysis of $J / \psi \rightarrow \pi^{+} \pi^{-} \pi^{0}, J / \psi \rightarrow K^{+} K^{-} \pi^{0}$ and $J / \psi \rightarrow K_{S}^{0} K^{ \pm} \pi^{\mp}$}

Only a preliminary result exists, to date, on a Dalitz-plot analysis of $J / \psi$ decays to $\pi^{+} \pi^{-} \pi^{0}$ [7]. While large samples of $J / \psi$ decays exist, some branching fractions remain poorly measured. BES III experiment has performed an angular analysis of $J / \psi \rightarrow K^{+} K^{-} \pi^{0}$. The analysis requires the presence of a broad $J^{P C}=1^{--}$state in the $K^{+} K^{-}$threshold region, which is interpreted as a multiquark state [8].

We study the following reactions:

$$
e^{+} e^{-} \rightarrow \gamma_{\mathrm{ISR}} \pi^{+} \pi^{-} \pi^{0}, \quad e^{+} e^{-} \rightarrow \gamma_{\mathrm{ISR}} K^{+} K^{-} \pi^{0}, \quad e^{+} e^{-} \rightarrow \gamma_{\mathrm{ISR}} K_{S}^{0} K^{ \pm} \pi^{\mp}
$$



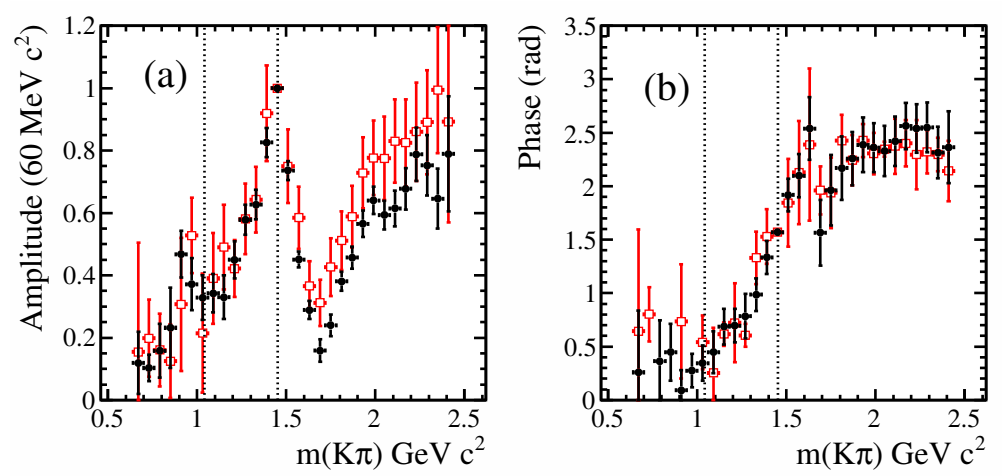

Figure 3. The $I=1 / 2 K \pi \mathcal{S}$-wave amplitude (a) and phase (b) from $\eta_{c} \rightarrow K_{S}^{0} K^{+} \pi^{-}$(solid (black) points) and $\eta_{c} \rightarrow K^{+} K^{-} \pi^{0}$ (open (red) points); only statistical uncertainties are shown. The dotted lines indicate the $K \eta$ and $K \eta^{\prime}$ thresholds.

where $\gamma_{\mathrm{ISR}}$ indicate the (mostly undetected) ISR photon. We compute $M_{\mathrm{rec}}^{2} \equiv\left(p_{e^{-}}+p_{e^{+}}-p_{h^{+}}-p_{h^{-}}-\right.$ $\left.p_{h^{0}}\right)^{2}$, where $h$ indicates the three hadrons in the final states. This quantity should peak near zero for ISR events. We select events in the ISR region by requiring $\left|M_{\mathrm{rec}}^{2}\right|<2 \mathrm{GeV}^{2} / \mathrm{c}^{4}$ for the reactions involving a $\pi^{0}$ and $\left|M_{\mathrm{rec}}^{2}\right|<1.5 \mathrm{GeV}^{2} / \mathrm{c}^{4}$ for the reaction with a $K_{S}^{0}$. We fit the mass spectra using the Monte Carlo resolution functions described by a Crystal Ball+Gaussian functions. We obtain 19560 \pm 164 events for $J / \psi \rightarrow \pi^{+} \pi^{-} \pi^{0}$ with $(91.3 \pm 0.2) \%$ purity, $2002 \pm 48$ for $J / \psi \rightarrow K^{+} K^{-} \pi^{0}$ with $(88.8 \pm 0.7) \%$ purity, and $3694 \pm 64$ with $93.1 \pm 0.4$ purity for $J / \psi \rightarrow K_{S}^{0} K^{ \pm} \pi^{\mp}$. The efficiency is mapped and fitted on the $\left(m\left(h^{+} h^{-}\right), \cos \theta_{h}\right)$ plane, where $\theta_{h}$ is the $h^{+}$helicity angle in the $J / \psi$ rest frame. To obtain the measurements of the relative branching fractions, we correct yields by weighting each event by the inverse of the efficiency and perform background subtraction by assigning negative weights to events the $J / \psi$ sidebands regions. We measure the ratio

$$
\mathcal{R}_{1}=\frac{\mathcal{B}\left(J / \psi \rightarrow K^{+} K^{-} \pi^{0}\right)}{\mathcal{B}\left(J / \psi \rightarrow \pi^{+} \pi^{-} \pi^{0}\right)}=0.120 \pm 0.003 \text { (stat) } \pm 0.009 \text { (sys) }
$$

where many systematic uncertainties cancel out due to the similar event topology of the $J / \psi$ decay modes. The PDG reports $\mathcal{B}\left(J / \psi \rightarrow \pi^{+} \pi^{-} \pi^{0}\right)=(2.11 \pm 0.07) \times 10^{-2}$, while the branching fraction $\mathcal{B}\left(J / \psi \rightarrow K^{+} K^{-} \pi^{0}\right)$ has been measured by Mark II [9] using 25 events, to be $(2.8 \pm 0.8) \times 10^{-3}$. These values give a ratio $\mathcal{R}_{1}^{P D G}=0.133 \pm 0.038$, in agreement with our measurement. Using a similar procedure and correcting correcting for unseen $K_{s}^{0}$ decay modes, we compute the relative branching fraction

$$
\mathcal{R}_{2}=\frac{\mathcal{B}\left(J / \psi \rightarrow K_{s}^{0} K^{ \pm} \pi^{\mp}\right)}{\mathcal{B}\left(J / \psi \rightarrow \pi^{+} \pi^{-} \pi^{0}\right)}=0.265 \pm 0.005 \text { (stat) } \pm 0.021 \text { (sys). }
$$

The branching fraction $\mathcal{B}\left(J / \psi \rightarrow K_{S}^{0} K^{ \pm} \pi^{\mp}\right)$ has been measured by Mark I [10], using 126 events, to be $(26 \pm 7) \times 10^{-4}$. Using the above measurements we obtain an estimate of $\mathcal{R}_{2}^{P D G}=0.123 \pm 0.033$, which deviates by $3.6 \sigma$ from our measurement.

\section{$5 \mathrm{~J} / \psi \rightarrow \pi^{+} \pi^{-} \pi^{0}$ Dalitz plot analysis}

The Dalitz plot for $J / \psi \rightarrow \pi^{+} \pi^{-} \pi^{0}$ is shown in Fig. 4 and is dominated by three $\rho(770) \pi$ contributions. We perform a Dalitz plot analysis using the isobar model with amplitudes described by Zemach 


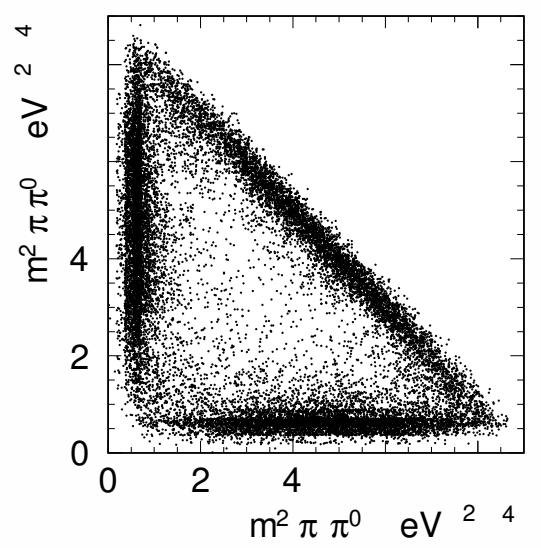

Figure 4. $J / \psi \rightarrow \pi^{+} \pi^{-} \pi^{0}$ Dalitz plot.

tensors $[11,12]$ and the Veneziano model [13]. The results from the Dalitz analysis are tabulated in Table 2 and fit projections are shown in fig. 5. The Veneziano model deals with trajectories rather
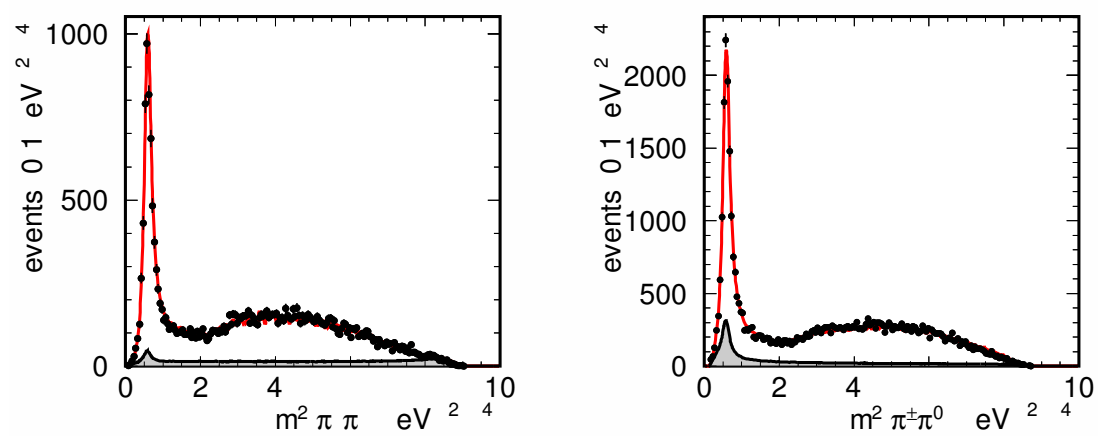

Figure 5. (left) $m^{2}\left(\pi^{+} \pi^{-}\right)$and (right) $m^{2}\left(\pi^{ \pm} \pi^{0}\right)$ for $J / \psi \rightarrow \pi^{+} \pi^{-} \pi^{0}$. Shaded is the background interpolated from $J / \psi$ sidebands.

than single resonances. The complexity of the model is related to $n$, the number of Regge trajectories included in the fit which requires $n=7$, described by 19 free parameters.

Figure 8 (a) shows the combinatorial $\cos \theta_{\pi}$ helicity angle vs. $m(\pi \pi)$. Figure 8 also shows the $m(\pi \pi)$ mass projection for $\left|\cos \theta_{\pi}\right|<0.2$ for the isobar model fit (b) and Veneziano model (c). The helicity cut removes the $\rho$ reflections enhancing the true $\rho$ signals. The fitted fractions and phases from the Dalitz analyses are summarized in Table 2. The two models give almost similar data representation, but different fractions. 
Table 2. Results from the Dalitz-plot analysis of the $J / \psi \rightarrow \pi^{+} \pi^{-} \pi^{0}$ channel. When two uncertainties are given, the first is statistical and the second systematic. The error on the amplitude is only statistical.

\begin{tabular}{lccc|c}
\hline Final state & Amplitude & Isobar fraction $(\%)$ & Phase (radians) & Veneziano fraction $(\%)$ \\
\hline$\rho(770) \pi$ & 1. & $114.2 \pm 1.1 \pm 2.6$ & 0. & $133.1 \pm 3.3$ \\
$\rho(1450) \pi$ & $0.513 \pm 0.039$ & $10.9 \pm 1.7 \pm 2.7$ & $-2.63 \pm 0.04 \pm 0.06$ & $0.80 \pm 0.27$ \\
$\rho(1700) \pi$ & $0.067 \pm 0.007$ & $0.8 \pm 0.2 \pm 0.5$ & $-0.46 \pm 0.17 \pm 0.21$ & $2.20 \pm 0.60$ \\
$\rho(2150) \pi$ & $0.042 \pm 0.008$ & $0.04 \pm 0.01 \pm 0.20$ & $1.70 \pm 0.21 \pm 0.12$ & $6.00 \pm 2.50$ \\
$\omega(783) \pi^{0}$ & $0.013 \pm 0.002$ & $0.08 \pm 0.03 \pm 0.02$ & $2.78 \pm 0.20 \pm 0.31$ & \\
$\rho_{3}(1690) \pi$ & & & & $0.40 \pm 0.08$ \\
\hline Sum & & $127.8 \pm 2.0 \pm 4.3$ & & $142.5 \pm 2.8$ \\
$\chi^{2} / v$ & $687 / 519=1.32$ & & $596 / 508=1.17$ \\
\hline
\end{tabular}
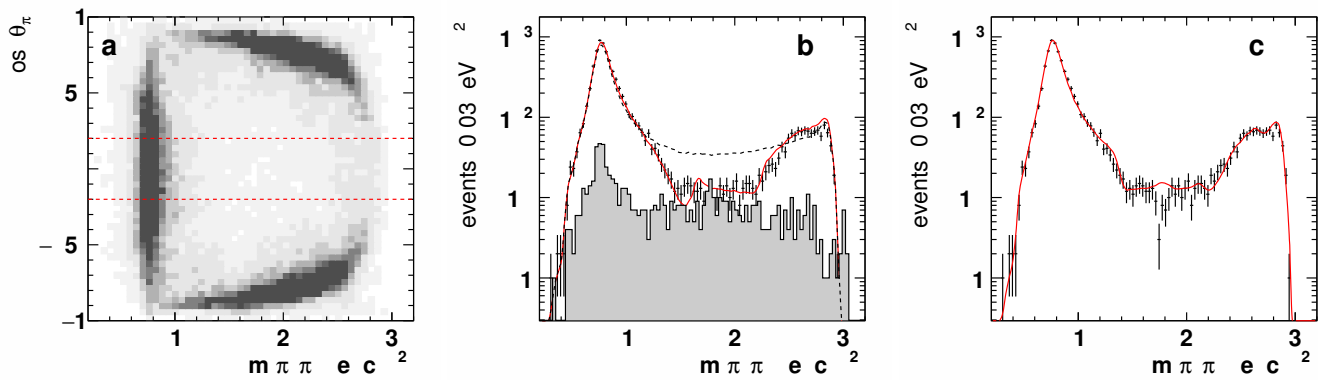

Figure 6. (a) Binned scatter diagram of $\cos \theta_{\pi_{3}} v s m\left(\pi_{1} \pi_{2}\right)$. (b), (c) $\pi \pi$ mass projection in the $\left|\cos \theta_{\pi}\right|<0.2$ region for all the three $\pi \pi$ charge combinations. The horizontal lines in (a) indicate the $\cos \theta_{\pi}$ selection. The dashed line in (b) is the result from the fit with only the $\rho(770) \pi$ amplitude. The fit in (b) uses the isobar model and the shaded histogram shows the background distribution estimated from the $J / \psi$ sidebands. The fit in (c) uses the Veneziano model.

\section{$6 \mathrm{~J} / \psi \rightarrow K^{+} K^{-} \pi^{0}$ and $J / \psi \rightarrow K_{S}^{0} K^{ \pm} \pi^{\mp}$ Dalitz plot analyses}

We perform a Dalitz-plot analysis of $J / \psi \rightarrow K^{+} K^{-} \pi^{0}$ in the $J / \psi$ signal region. Figure 7(left) shows the Dalitz plot for the $J / \psi$ signal region and Fig. 8(left) shows the Dalitz plot projections. We observe that the decay is dominated by the $K^{*}(892)^{ \pm} K^{\mp}$ amplitude. We also observe a diagonal band which we tentatively attribute to the $\rho(1450)^{0} \pi^{0}$ amplitude. We fit the $J / \psi \rightarrow K^{+} K^{-} \pi^{0}$ Dalitz plot using the isobar model. The results from the fit are given in Table 3 and fit projections are shown in Fig. 8(left). The Dalitz analysis is describing well the $K^{+} K^{-}$threshold region and therefore we assign the broad enhancement in the $K^{+} K^{-}$mass spectrum to the presence of the $\rho(1450)$ resonance: the present data do not require the presence of an exotic contribution.

We perform a similar Dalitz plot analysis of $J / \psi \rightarrow K_{S}^{0} K^{ \pm} \pi^{\mp}$ in the $J / \psi$ signal region. Figure 7(right) shows the Dalitz plot for the $J / \psi$ signal region and Fig. 8(right) shows the Dalitz plot projections. We fit the $J / \psi \rightarrow K_{S}^{0} K^{ \pm} \pi^{\mp}$ Dalitz plot using the isobar model. The results from the best fit are summarized in Table 4 . The decay is dominated by the $K^{*}(892) \bar{K}, K_{2}^{*}(1430) \bar{K}$ and $\rho(1450)^{ \pm} \pi^{\mp}$ 

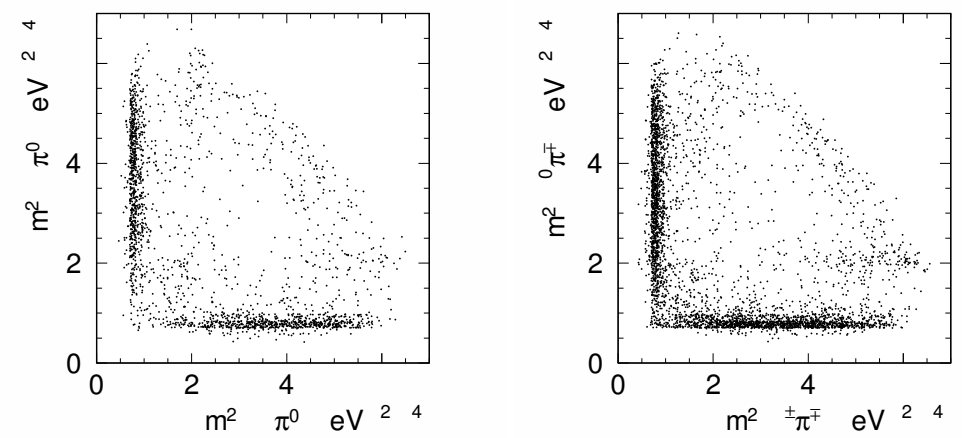

Figure 7. (left) $J / \psi \rightarrow K^{+} K^{-} \pi^{0}$ and (right) $J / \psi \rightarrow K_{S}^{0} K^{ \pm} \pi^{\mp}$ Dalitz plots.
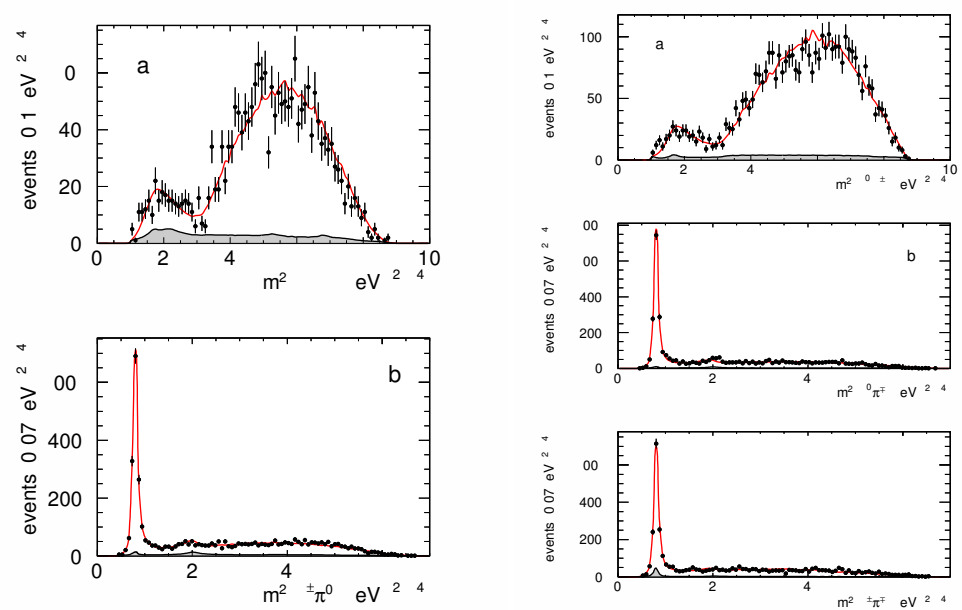

Figure 8. (left) Dalitz plot projections with fit results for $J / \psi \rightarrow K^{+} K^{-} \pi^{0}$. (right) Dalitz plot projections with fit results for $J / \psi \rightarrow K_{S}^{0} K^{ \pm} \pi^{\mp}$. Shaded is the background interpolated from $J / \psi$ sidebands.

amplitudes with a smaller contribution from the $K_{1}^{*}(1410) \bar{K}$ amplitude. Also in this case we assign the broad enhancement observed in the $K_{S}^{0} K^{ \pm}$mass projection to the presence of the $\rho(1450)^{ \pm}$resonance.

In the Dalitz-plot analysis of $J / \psi \rightarrow K^{+} K^{-} \pi^{0}$, the data are consistent with the observation of the decay $\rho(1450)^{0} \rightarrow K^{+} K^{-}$. This allows a measurement of its relative branching fraction to $\rho(1450)^{0} \rightarrow \pi^{+} \pi^{-}$. We obtain:

$$
\frac{\mathcal{B}\left(\rho(1450)^{0} \rightarrow K^{+} K^{-}\right)}{\mathcal{B}\left(\rho(1450)^{0} \rightarrow \pi^{+} \pi^{-}\right)}=0.307 \pm 0.084 \text { (stat) } \pm 0.082 \text { (sys) }
$$


Table 3. Results from the Dalitz-plot analysis of the $J / \psi \rightarrow K^{+} K^{-} \pi^{0}$ signal region. When two uncertainties are given, the first is statistical and the second systematic.

\begin{tabular}{lcr}
\hline Final state & fraction $(\%)$ & phase (radians) \\
\hline$K^{*}(892)^{ \pm} K^{\mp}$ & $92.4 \pm 1.5 \pm 3.4$ & 0. \\
$\rho(1450)^{0} \pi^{0}$ & $9.3 \pm 2.0 \pm 0.6$ & $3.78 \pm 0.28 \pm 0.08$ \\
$K_{1}^{*}(1410)^{ \pm} K^{\mp}$ & $2.3 \pm 1.1 \pm 0.7$ & $3.29 \pm 0.26 \pm 0.39$ \\
$K_{2}^{*}(1430)^{ \pm} K^{\mp}$ & $3.5 \pm 1.3 \pm 0.9$ & $-2.32 \pm 0.22 \pm 0.05$ \\
\hline Total & $107.4 \pm 2.8$ & \\
$\chi^{2} / v$ & $132 / 137=0.96$ & \\
\hline
\end{tabular}

Table 4. Results from the Dalitz-plot analysis of the $J / \psi \rightarrow K_{S}^{0} K^{ \pm} \pi^{\mp}$ signal region. When two uncertainties are given, the first is statistical and the second systematic.

\begin{tabular}{lrr}
\hline Final state & \multicolumn{1}{c}{ fraction $(\%)$} & \multicolumn{1}{c}{ phase (radians) } \\
\hline$K^{*}(892) \bar{K}$ & $90.5 \pm 0.9 \pm 3.8$ & 0. \\
$\rho(1450)^{ \pm} \pi^{\mp}$ & $6.3 \pm 0.8 \pm 0.6$ & $-3.25 \pm 0.13 \pm 0.21$ \\
$K_{1}^{*}(1410) \bar{K}$ & $1.5 \pm 0.5 \pm 0.9$ & $1.42 \pm 0.31 \pm 0.35$ \\
$K_{2}^{*}(1430) \bar{K}$ & $7.1 \pm 1.3 \pm 1.2$ & $-2.54 \pm 0.12 \pm 0.12$ \\
\hline Total & $105.3 \pm 3.1$ & \\
\hline$\chi^{2} / v$ & $274 / 217=1.26$ & \\
\hline
\end{tabular}

\section{Ackowledgements}

This work was supported (in part) by the U.S. Department of Energy, Office of Science, Office of Nuclear Physics under contract DE-AC05-06OR23177.

\section{References}

[1] J. P. Lees et al. [BaBar Collaboration], Phys. Rev. D 89, no. 11, 112004 (2014)

[2] Charge conjugation is implied through all this work.

[3] S. Eidelman et al. [Particle Data Group], Phys. Lett. B 592, no. 1-4, 1 (2004).

[4] E. M. Aitala et al. [E791 Collaboration], Phys. Rev. D 73, 032004 (2006) Erratum: [Phys. Rev. D 74, 059901 (2006)]

[5] D. Aston et al., Nucl. Phys. B 296, 493 (1988).

[6] K. M. Watson, Phys. Rev. 88, 1163 (1952).

[7] L. Chen et al. [MARK-III Collaboration], SLAC-PUB-5674.

[8] M. Ablikim et al., (BESIII Collaboration), Phys. Lett. B 710, 594 (2012).

[9] M.E.B. Franklin et al., (Mark II Collaboration) Phys. Rev. Lett. 51, 963 (1983).

[10] F. Vannucci et al., (Mark I Collaboration) Phys. Rev. D 151814 (1977).

[11] C. Zemach, Phys. Rev. 133, B1201 (1964).

[12] C. Dionisi et al., Nucl. Phys. B 169, 1 (1980).

[13] A. P. Szczepaniak, M.R. Pennington, Phys. Lett. B 737, 283 (2014). 